Revista Signos 2010, 43(73) 307-331

\title{
El papel de la reformulación en los intercambios adulto-niño: Un estudio de caso
}

\author{
María Luisa Silva \\ Centro de Investigación Interdisciplinario \\ en Psicología y Matemática Experimental \\ Argentina
}

Resumen: Este trabajo analiza y compara la presencia y distribución de los procedimientos de reformulación utilizados por un niño de cinco años y dos adultos en dos tipos diferentes de interacción comunicativa: una entrevista semidirigida y el momento escolar denominado 'comentario grupal/ ronda'. Los resultados indican que, pese a que son diferentes sujetos, los dos adultos comparten ciertas características en el uso de los procedimientos de reformulación. El niño, en cambio presenta diferencias notables en el uso de estos procedimientos según los tipos de texto. En virtud del marco teórico (Jefferson, 1983; Gülich \& Kotschi, 1997; Cole, 1999; Silva, 2007), estas diferencias se pueden atribuir a las posibilidades que cada tipo de interacción plantea para que los participantes monitoreen el texto en gestión.

Palabras Clave: Reformulación, competencia comunicativa, lenguaje infantil, análisis de la conversación.

Recibido: 11-XII-2008 Aceptado: 5-I-2010
Correspondencia: María Luisa Silva (mluisa@filo.uba.ar). Centro de Investigación Interdisciplinario en Psicología y Matemática Experimental. J. D. Perón 2158 cp 1040, Buenos Aires, Argentina. 


\title{
Repair role in adult-child conversational exchanges: A case study
}

\begin{abstract}
This article analyzes and compares the use and distribution of repairs employed by a fiveyear-old child and two adults in two different communicative interactions: an interview and in a kindergarten event named 'circle'. The results indicate that, although adults are different individuals, both of them share some certain characteristics of repair moves usage. In contrast, child repair usages exhibit important differences according to each type text. Considering theoretical assumptions (Cole, 1999; Gülich \& Kotschi, 1997; Jefferson, 1983; Silva, 2007), these variations can be attributed to the opportunities that each type of interaction poses for participants in order to monitor the text production process on line.
\end{abstract}

Key Words: Reformulation, communicative competence, child language, conversation analysis.

\section{INTRODUCCIÓN}

En el presente trabajo se analizan y comparan los procedimientos de reformulación actualizados por un mismo niño y dos adultos, interlocutores del niño, en dos diferentes situaciones comunicativas.

La gestión y características de las reformulaciones han sido estudiadas inicialmente en el marco de los estudios sobre la interacción conversacional (Schegloff, Jefferson \& Sacks, 1977; Gumperz, 1982; Jefferson, 1983). Los aportes analíticos de estos trabajos han sido incorporados y complejizados por otras teorías (Gülich \& Kotschi, 1987, 1997; Pomeranz \& Fehr, 2001). De esta forma se han estudiado una gran variedad de interacciones, entre ellas las interacciones entre niños y adultos. El análisis de este último tipo de interacciones ha permitido reconocer como una de las características distintivas el hecho de que en estas el adulto crea entornos en los que negocia, comparte o cede al niño el control del texto en gestión (Silva, 2007).

Este trabajo procura, a partir del análisis comparativo de los procedimientos de reformulación utilizados por un niño y dos adultos en dos tipos de interacciones, explorar y describir las características que poseen las reformulaciones de niños y adultos en tanto marcas del ejercicio de la actividad de control sobre el texto cooperativo.

\section{Marco teórico}

Los estudios de los analistas de la conversación comprenden esta interacción comunicativa como un tipo particular de relación social que genera un producto: el texto. En los textos los participantes modelan su intervención y aportes, dejando ciertas 'marcas' de esa actividad. 
Consecuentemente se comprende la formulación textual como una tarea que conlleva esfuerzo cognitivo e insume tiempo, pues involucra tomar decisiones para resolver problemas de formulación. En este sentido la formulación se asemeja a un complejo proceso de resolución de problemas (Newell \& Simon, 1972). La disposición y orquestación de recursos lingüísticos, prosódicos, gestuales y proxémicos se consideran 'marcas' que nos permiten inferir características de la organización social que se reflejan en el intercambio comunicativo.

Al analizar la actividad de formulación, Schegloff, Jefferson y Sacks (1977) identificaron un conjunto de recursos que hablantes y oyentes utilizan para resolver problemas de mutua comprensión. Clasificaron estos usos como procedimientos de reformulación (repair). En un procedimiento de reformulación, el hablante reconoce un segmento de discurso que puede resultar potencialmente problemático para la comprensión mutua y lo reformula en función de las posibilidades de comprensión que le adjudica a su interlocutor. Las expresiones que resultan problemáticas pueden serlo porque el hablante las juzga conceptualmente imprecisas, vagas o ambigua 0 , incluso, porque al articularlas deficientemente considera que pueden resultar confusas para el oyente.

Jefferson (1983) describe la ocurrencia del proceso de reformulación en función de la emergencia de tres rasgos: a) la progresión temática que se desarrolla en el intercambio conversacional se discontinúa y el proceso de reformulación se convierte en la principal actividad de los participantes del intercambio; b) generalmente se acompaña el proceso de reformulación con otras actividades (gestos, especialmente) que ‘colaboran' con la reformulación; y c) existe una secuencia de enunciados $(X, Y, Z)$, que conforman la reformulación, que un hablante produce en relación con las expresiones de otro interlocutor o a las de sí mismo. Por su parte, Schegloff, Jefferson y Sacks (1977) clasifican los actos de reformulación en virtud de la relación de las expresiones con los participantes del intercambio. Cuando el acto relaciona dos expresiones del mismo hablante los denominan Autorreformulación, en cambio, si la expresión comprende los actos que vinculan las expresiones de diferentes hablantes se denomina 'Heterorreformulación'.

La Teoría de Acciones de Producción Discursiva (Gülich \& Kotschi, 1987, 1997; Ciapuscio, 1997) adopta los postulados básicos de los estudios conversacionales para describir, analizar y clasificar los diferentes procedimientos de reformulación para elaborar una teoría de los procesos de composición textual. Así, diferencian las ‘huellas de la formulación’ según se traten de: procedimientos de verbalización, procedimientos de tratamiento o procedimientos de evaluación (o calificación metadiscursiva) (Gülich \& Kotschi, 1987, 1997). Los procedimientos de verbalización son fenómenos que reflejan la puesta en texto de los contenidos cognitivos, se consideran 
típicos del habla, interrumpen la sucesión sintagmática y se clasifican según el nivel lingüístico de ocurrencia; diferenciándose, entonces, los que afectan la realización fonética, los que involucran procesos del nivel morfo-léxico, los del nivel léxico- semántico, los del sintáctico o aquellos que comprenden al nivel pragmático. Entre los procedimientos de verbalización se encuentran: las autorreparaciones, los fenómenos de duda, las expresiones incompletas, los falsos comienzos, las repeticiones de sílabas y las repeticiones de palabras.

Los procedimientos de tratamiento, en cambio, comprenden secuencias en las cuales un hablante refiere a un segmento previo con otra expresión. Su utilización implica que el hablante ha realizado esta operación después de haber evaluado la expresión previa como insuficiente, inapropiada o insatisfactoria. Dentro de este grupo hallamos repeticiones, cambios, reformulaciones, síntesis o expansiones de expresiones previas.

Considerando la relación entre el procedimiento de tratamiento y la expresión 'tratada' o previa es posible clasificar los procedimientos de tratamiento según grados de afinidad, planteando una relación que opere en un continuo entre los polos de la equivalencia y de la divergencia. De esta manera, en la medida que un hablante evalúe el grado de validación que le otorga a una expresión 'tratada' realizará selecciones dentro de ese continuo: un grado alto de validación conllevaría el uso de una repetición y el grado alto de invalidación una corrección.

Los procedimientos de evaluación metadiscursiva y de comentario le permiten al hablante referir y caracterizar diferentes problemas acaecidos durante la producción discursiva. Estos procedimientos, a diferencia de los de tratamiento que típicamente resuelven la perturbación, al designar ciertos problemas de comunicación representan la manifestación explícita del constante monitoreo cognitivo de los hablantes (Gülich \& Kotschi, 1987, 1997; Ciapuscio, 1997).

La diferencia esencial que permite distinguir los fenómenos de verbalización con respecto a los de tratamiento y calificación es que los primeros ocurren en forma simultánea con la producción de las emisiones; en cambio, los últimos, ocurren como actividades adicionales.

Por otro lado, Gülich y Kotschi $(1987,1997)$ retoman las categorías de ‘Autorreformulación’ y 'Heterorreformulación' (Sacks, Schegloff \& Jefferson, 1977) para distinguir los actos de composición textual en virtud de los participantes del intercambio. Así conservan la denominación de 'Autorreformulación' para los procedimientos que vinculan dos expresiones de un mismo hablante. En cambio, a la 'Heterorreformulación' la denominan 'Reformulación externa'.

Aunque estos conceptos surgieron del análisis de intercambios cara a cara espontáneos entre adultos, se ha demostrado su funcionalidad tanto para el análisis del desarrollo de las competencias conversacionales infantiles (Garvey, 1977; Tomasello, Farrar \& Dines, 1983; Anselmi, 
Tomasello \& Acunzo, 1986; Lloyd, 1991) como también de la dinámica de los intercambios docente-alumnos en el contexto áulico (Erickson, 1996; Macbeth, 2004; Rosemberg \& Silva, 2009; entre otros). Así, se ha reconocido que los niños pueden reformular expresiones a edades tempranas (Garvey, 1977) y que exhiben diferencias en el ejercicio de la actividad, por ejemplo, en relación con los interlocutores (Tomasello, Farrar \& Dines, 1983) o con los tipos de problemas que identifican (Schwe \& Markman, 1997).

En cuanto a los trabajos sobre la reformulación en la interacción docente-niños se han reconocido y clasificado los procedimientos y además se ha procurado explicar el uso diferencial de los mismos así como su utilidad para el aprendizaje (Erickson, 1996; Macbeth, 2004; Rosemberg \& Silva, 2009).

En lo que concierne al análisis de los procedimientos que los niños utilizan en los jardines de infantes cuando se deben comentar eventos pretéritos, el análisis socio-interaccional también ha realizado importantes aportes. A partir del trabajo pionero de Michaels (1988), que ha permitido comprender cómo las diferencias culturales generan 'estilos' de interacción muchas veces poco comprendidos por los docentes, se ha podido configurar un cuadro descriptivo exhaustivo de la particular dinámica del momento de comentario grupal (Erickson, 1996, 2004; Rosemberg, 2002; Rosemberg \& Manrique, 2004, 2005).

No obstante el importante desarrollo analítico y teórico reseñado, consideramos que aún resta abordar sistemáticamente la comparación y el análisis de cómo los niños utilizan las reformulaciones comparando los intercambios que ocurren en instancias escolares (formales) con los que ocurren en situaciones no escolares.

En función de atender a esta última cuestión, este trabajo suma a los aportes teóricos, analíticos y metodológicos mencionados, la perspectiva presentada en Silva (2007) en la que se consideran a los procedimientos de reformulación como instancias particulares del proceso por el cual una actividad psicológica se internaliza a partir de la mediación simbólica. Los procesos de reformulación activan la dinámica del desarrollo en la medida en que se originan en la interacción de los sujetos. Se elaboró un sistema de categorías en la que se distinguen los procesos de reformulación en tanto su uso depende del grado de apropiación de la actividad (ejercicio frecuente de la práctica), y de la existencia de una percepción innovadora que ocasiona el uso del recurso. El uso de cualquier procedimiento de reformulación supone, entonces, que los participantes se han apropiado de variadas situaciones de intercambio y por ello, en virtud de la relación establecida entre actividad de monitoreo y actos de formulación es posible comprender estos últimos como huellas de la interacción y de la gestión del control, y, también, como instancias de desarrollo de funciones cognitivas. La diferencia entre los usos 
adultos y los infantiles radica en que el adulto ha tenido posibilidad de internalizar variadas y múltiples actividades de interacción y, por ello, dispone de una gama más variada de recursos para monitorear problemas comunicativos, y puede activar con menor esfuerzo los procesos que le permiten resolverlos. El niño, en cambio, solo puede monitorear cierto tipo de interacciones, las más ritualizadas y en las que ha participado con mayor frecuencia. Esta diferencia nos permite comprender por qué distintos adultos pueden presentar características comunes en su patrón comunicativo, en cambio los niños pueden presentar diferencias notables en su patrón de uso de recursos según se consideren diferentes factores, por ejemplo: el formato, el interlocutor, la tarea, etc. (Silva, Plana \& Borzone, 2007).

\section{Metodología}

En el presente trabajo analizaremos y compararemos las reformulaciones que dos participantes, un niño de 5,9 años y dos adultos, introducen en dos intercambios conversacionales. Estos textos, generados por el esfuerzo de construcción dialógica, cooperativa, entre el niño y los adultos, se pueden considerar ejemplos típicos del proceso de apropiación de uso del lenguaje (Vygotsky, 1961, 1993; Cole, 1999; Silvestri, 1999; Silva, 2007).

Ambos intercambios surgieron de instancias de interacción asimétricas: entre los adultos y el niño existen considerables diferencias de capital simbólico y lingüístico. Uno de los intercambios se produjo en el momento de aprendizaje denominado 'comentario grupal/ronda de cierre', una instancia de aprendizaje formal, muy ritualizada, en la que el adulto ejerce la función de docente. El otro segmento pertenece a una entrevista individual semidirigida. Ambos intercambios se registraron dentro de la sala del jardín de infantes a la que el niño concurre, por lo que el ámbito de elicitación reafirma también la relación asimétrica.

El jardín de infantes se encuentra en la ciudad de Buenos Aires, República Argentina, y atiende a una población de nivel socioeconómico bajo; mayoritariamente de nacionalidad peruana, boliviana y paraguaya. Los docentes comentan que los niños presentan una compleja problemática en cuanto al uso de recursos lingüísticos ${ }^{1}$. Los dos adultos, en cambio, pertenecen a un nivel socioeconómico medio y ambos poseen más de 15 años de educación.

Hemos denominado Texto 1 (T1) a la transcripción de la entrevista semidirigida y Texto 2 (T2) al de la transcripción del momento de intercambio grupal. En el T1 el entrevistador, a partir de indagar sobre tópicos infantiles significativos (el cumpleaños, accidentes leves, paseos, etc.), procuraba que el niño produjera narrativas autónomas. El T2 es la elicitación del momento de intercambio grupal, un evento muy rutinario en la jornada del jardín de infantes. En este tipo 
de interacción cada niño debe ser capaz de narrar individualmente eventos pasados, en tanto los otros niños constituyen el auditorio y deben realizar una 'escucha atenta'. El docente es el 'moderador' del intercambio: selecciona al niño que toma la palabra, colabora en la construcción del texto, pauta las intervenciones, controla la coherencia y duración del relato y se preocupa porque los demás niños escuchen atentamente el relato de su compañero. El texto analizado consiste en una serie de intercambios ocurridos entre el niño y la maestra a posteriori de un momento de juego libre en los rincones.

Entre las dos secuencias existe una diferencia de duración: la entrevista comprende unos 10 minutos, en tanto que el segmento de intercambio grupal ronda los 7 minutos. Asimismo esa diferencia de duración se manifiesta en la cantidad de turnos. En el T1 se analizan 76 turnos de habla con 76 enunciados. De ese total, 43 han sido proferidos por el niño y 33 por el adulto. En el T2 se analizan 19 turnos de habla, con 23 enunciados, de los cuales 11 le corresponden al niño y los restantes al adulto.

\subsection{Transcripción y análisis}

Se transcribieron los textos según las pautas desarrolladas por los analistas de la conversación (Jefferson, 2004). Para el análisis se seleccionaron y analizaron todas las secuencias que incluían reformulaciones. Para identificar el comportamiento comunicativo de cada uno de los participantes se compararon los diferentes tipos de procedimientos de reformulación, así como los contextos de aparición en cada uno de los textos. Se consideraron como medidas las frecuencias relativas de aparición y dos índices elaborados para comparar la relación entre la cantidad de procedimientos y la cantidad de enunciados: el Índice de Concentración de Reformulaciones (ICR) y el Índice de Concentración de Procedimientos de Reformulación (ICPR). Estos índices se calcularon a partir de considerar la relación entre la cantidad de procedimientos y la cantidad de enunciados. De esta forma cuando el ICR o el ICPR se aproxime a 1 se considera como la expresión de grado de concentración máxima, en la que en todos los enunciados se encuentran reformulaciones. Para diseñar estos índices se ha tomado como modelo el cálculo del Índice de Complejidad Sintáctica (Hunt, 1970; Véliz, 1999).

En cuanto a las categorías de análisis se consideraron la clasificación de procedimientos de reformulación en virtud de los participantes del intercambio: ‘Autorreformulación' y 'Reformulación externa' (Gülich \& Kotschi, 1987, 1997) y según el tipo de procedimiento: 'Procedimientos de verbalización', ‘Procedimientos de tratamiento’ o ‘Procedimientos de evaluación' (Gülich \& Kotschi, 1987, 1997; Ciapuscio, 1997). 


\section{Resultados}

En la Tabla 1 se compara el uso de las reformulaciones en los dos segmentos de textos considerados.

Tabla 1. Frecuencia relativa e Índice de Concentración de Reformulaciones según los participantes y los tipos de texto.

\begin{tabular}{|l|c|c|c|c|}
\hline & \multicolumn{2}{|c|}{ Frecuencia } & \multicolumn{2}{c|}{ Índice de Concentración de Reformulaciones } \\
\hline Participantes & $\mathrm{T} 1$ & $\mathrm{~T} 2$ & $\mathrm{~T} 1$ & $\mathrm{~T} 2$ \\
\hline Niño & 58,13 & 80 & 0,6 & 1 \\
\hline Adulto & 73,10 & 60 & 0,83 & 0,9 \\
\hline
\end{tabular}

La Tabla 1 nos permite observar que, pese a las diferentes demandas comunicativas que cada tipo de interacción plantea, los adultos utilizan un porcentaje similar de procedimientos de reformulación ( $73,10 \%$ y $60 \%$ en $\mathrm{T} 1$ y $\mathrm{T} 2$, respectivamente); en cambio la diferencia en el caso del niño resulta considerable $(58,13 \%$ y $80 \%$, T1 y T2, respectivamente).

Este patrón se observa más claramente en el caso del Índice de Concentración de Reformulaciones (ICR). En efecto observamos que los adultos presentan un ICR muy semejante en los dos Textos (0,83 y 0,9, para T1 y T2, respectivamente), en cambio la diferencia de ICR en el caso del niño resulta muy importante $(0,6$ y 1 , para $\mathrm{T} 1$ y $\mathrm{T} 2$, respectivamente).

Pareciera que, mientras que los adultos observan cierto patrón de comportamiento para la introducción de reformulaciones, el niño reformula con mayor frecuencia en el T2 que en el T1.

Ahora bien, si las reformulaciones son un índice del ejercicio de la función de control sobre el texto en gestión (Silva, 2007), las diferencias observadas nos permiten hipotetizar que, posiblemente, la función de monitoreo se actualice en forma diferente en el momento de ronda de lo que sucede en la entrevista. En efecto, pese a que ambas interacciones se han descripto como típicas en cuanto al carácter asimétrico de la relación entre los participantes, en el T2 el adulto parece ceder un poco más el control del texto en gestión. Esto significa que el niño en el momento de 'compartir', en tanto se encuentra en condiciones de asumir la responsabilidad compartida del monitoreo, puede introducir más reformulaciones no solo a su propia formulación sino también a las formulaciones del adulto.

Si esta hipótesis resultara descriptivamente adecuada deberíamos esperar que las reformulaciones del niño a las formulaciones del adulto ('Reformulaciones Externas') en el T2 fueran más frecuentes que en el T1. Para comprobar esta última presunción en la Tabla 2 se presenta el 
análisis de las reformulaciones del niño según se traten de 'Autorreformulaciones' o de 'Reformulaciones Externas'.

Tabla 2. Frecuencia relativa de tipos de reformulaciones según tipos de texto.

\begin{tabular}{|l|c|c|c|c|}
\hline Participantes & \multicolumn{2}{|c|}{ Reformulación Externa } & \multicolumn{2}{c|}{ Autorreformulación } \\
\hline & $\mathrm{T} 1$ & $\mathrm{~T} 2$ & $\mathrm{~T} 1$ & $\mathrm{~T} 2$ \\
\hline Niño & 0 & 80 & 100 & 20 \\
\hline
\end{tabular}

En la Tabla 2 se observa que en el T1 las reformulaciones del niño siempre son en relación a su propia participación (100\%), en el T2, en cambio, las reformulaciones del niño son mayoritariamente Reformulaciones Externas.

Esta diferencia en la distribución de los diferentes tipos de reformulaciones nos permite suponer que en el momento del comentario grupal ambos participantes construyen cooperativamente un texto para un auditorio y esto ocasiona que se negocie y controle la gestión del texto producido en el intercambio. En el T1, en cambio, el control del proceso de gestión descansa privativamente en el adulto, por lo que el niño se encuentra limitado para reformular las expresiones del adulto.

En este sentido, en el T1, se observa un fenómeno particular, que nos orienta acerca del tipo de actividad que cada uno de los participantes asume.

[El entrevistador procura que el niño expanda la descripción de un objeto, tratando de obtener la narrativa del cumpleaños]

$22 \mathrm{ENT}^{2} .:$ ¿De gokku / del dragon boll?

23 Niño: Sí:

24 ENT.: ¿Con la forma de goku?

25 Niño: ((Silencio. Gesto de afirmación solo con la cabeza, gira la cabeza hacia donde estaban jugando sus compañeros, flexiona el cuerpo No mira al entrevistador y continúa mirando a sus compañeros, estira el brazo tratando de tomar un autito))

26 ENT.: ¿Vos la pedisste?

27 Niño: ((Silencio Gesto afirmativo con la cabeza se agacha para tomar un autito)) 28 ENT.: ¿Y qué hiciste/ con la piñata?

29 Niño: ((Se incorpora y mira a la entrevistadora)) La rompí y todos se agarraron// $<$ Yo me agarré> una pistola grande ((señala una dimensión ensanchando la distancia entre las palmas))/que era así de tres plazas / de agua //de agua / esa cayó de la piñata // Yo me LA- AGARRÉ

En esta secuencia se observa que cuando el adulto percibe la distracción del niño (ls. 25 y 27), intenta distanciarse del tópico inicialmente planteado (ls. 26 y 28). Posiblemente el entrevis- 
tador ha considerado que el niño se ha distraído porque, en los turnos precedentes respondía a la requisitoria mirando al entrevistador, sentado en una silla, mientras sus compañeros jugaban a su lado en el piso, armando una autopista. En ese momento, en cambio, el niño comienza a prestar atención a lo que hacen sus compañeros. Procurando que el niño continúe interesado en la situación de entrevista, el adulto introduce una variación en el tópico (l.26) que no resulta fructífera, aunque en 1.28 presenta otra que logra ese cometido.

Si bien la distracción del niño podría ser atribuida a cierta labilidad atencional, consideramos que dicha explicación no resulta adecuada, esencialmente porque en ningún otro segmento ocurre un fenómeno similar. Por otro lado, si la atención del niño fuera lábil y decayera a los dos minutos de entrevista ¿cómo podemos explicar que durante los restantes ocho minutos no ocurra un fenómeno semejante? En este sentido consideramos que lo que se observa en este segmento es el hecho de que en la entrevista (T1) ambos participantes asumen y se distribuyen roles: el adulto indaga y el niño informa. Esta situación permite comprender por qué el niño puede 'distraerse', pues el adulto es el único que asume la responsabilidad de realizar actividades de monitoreo sobre el texto. Dicha actividad de control supone que el adulto debe, para convocar la atención del niño, modificar el tópico como un movimiento estratégico para no descartar su meta comunicativa: obtener información. En este sentido, si consideramos los enunciados del entrevistador, observamos que las preguntas de 1.26 y 1.28 las realiza en un tono de voz más elevado que el de otras interrogaciones del texto, enfatizando el movimiento de semicadencia ascendente característico de la interrogación. Consideramos que con este movimiento el entrevistador modela su participación ofreciendo al niño pistas para que comprenda que ha intentado convocar su atención.

En el T2, en cambio, el adulto no ha hecho uso de recursos para convocar la atención del niño. Además en ese texto hallamos Reformulaciones Externas tanto del niño como del adulto. Suponemos que varios factores podrían explicar estos fenómenos: en la ronda los dos participantes interactúan en una clave de mayor simetría, el intercambio posee una duración mucho más breve que la entrevista y es una situación muy ritualizada.

Como hemos visto, el análisis comparativo de la reformulaciones nos permite conocer las diferentes posibilidades que poseen el niño y el adulto para ceder, compartir o negociar el monitoreo del texto en gestión; no obstante consideramos necesario complementar ese análisis con el análisis comparativo de las características específicas que asume cada uso de los procedimientos según los articulen los adultos o el niño. Por ello, a continuación, comparamos las Reformulaciones Externas del niño y las de los adultos.

En el T2 dos enunciados del niño funcionan como Reformulaciones Externas, es decir, reformu- 
laciones de enunciados previos del docente.

[El niño narra las acciones que realizó en el juego en los rincones]

01 Niño: $\quad$ Yo jugué a la masa

02 Docente: Vos jugaste con la masa y:/ ¿qué hiciste con la masa?

03 Niño: ¡PLAS<TILINA>!

04 Docente: Y:/ ¿qué hicimos con la plastilina?

05 Niño: La masa la estiré así: /y entonces le puse arriba (de) la caja// y: aparecieron números

06 Docente: Y aparecieron nú=:/ y aparecieron números: $0 .$. ¿los hiciste vos/ los números?

07 Niño: Solamente aparecieron números / porque la caja tenía números.

08 Docente: A:: mirá / ¡qué bárbaro!

09 Niño: $\quad$ Y esos números./ eran de reloj

10 Docente: A:: mirá::// Vos agarraste la plastilina /y presionasste /y aparecieron los números

11 Niño: $\quad$ Y la puse del otro lado /y la aplastó /y aparecieron los número

En el primer ejemplo el niño formula un concepto que es retomado en el turno siguiente por el docente ("masa", en l.1). Al escucharlo parece que el niño lo juzga inadecuado e introduce una corrección (Gülich \& Kotschi, 1997) modificando notablemente el tono y la velocidad (l.3, "plastilina"). En efecto, el cambio de tono y de velocidad otorgan, por contraste con los enunciados previos, un énfasis peculiar a ese enunciado. Por otro lado observamos que con esta reformulación el niño no solo corrige a la docente sino que, también, se corrige a sí mismo y, con ello, demuestra al auditorio que conoce y que considera que la distancia conceptual entre “masa” y "plastilina” es significativa y que el uso de un término léxico inapropiado, en ese momento del intercambio, ocasionaría una seria perturbación del texto en gestión. No obstante, como puede apreciarse en l.05, sigue refiriéndose a ese elemento como "masa".

En el otro ejemplo de Reformulación Externa del niño, a diferencia de la interacción previa, el docente repite parcialmente (Gülich \& Kotschi, 1997) la expresión del niño (l.06), pero no comprende el proceso que dio lugar al estampado de los números. Por ello en el segundo segmento del enunciado introduce una corrección, en el marco sintáctico de una disyunción ("y aparecieron números: 0... ¿los hiciste vos/ los números?”), Macbeth (2004). En l.07 el niño responde a la pregunta del docente, aunque, en realidad, reformula el texto que ambos realizan cooperativamente pues menciona la causa del proceso ("porque la caja tenía números"). Si el niño se limitara a responder y no tuviera en cuenta su propia meta comunicativa (describir el proceso de estampado), podría haber dicho únicamente "no hice los números". No obstante, posiblemente porque el niño carece del acervo léxico que le permitiría detallar el proceso 
que permitió el estampado de los números, no puede solucionar el problema de comprensión del docente. En efecto en l.08, el docente se limita a calificar a la actividad ("qué bárbaro"). Cuando el niño aclara la semejanza de los números a los que hace referencia con la forma de un reloj, el docente puede iniciar la aclaración del procedimiento de estampado (l.10). Consideramos que el docente ha podido realizar esta acción discursiva porque la pista que le ofreció el niño le permitió recordar que en el rincón donde este había jugado se hallaba una caja con un juguete que servía para moldear plastilina. Pese a esto, en contraste con la situación analizada previamente, en este caso existe un grave problema de comprensión entre los interlocutores que a su vez repercute en el texto en gestión, pues ninguno de los participantes ha expandido mucho la descripción para que el auditorio comprenda cómo se produjo el proceso de estampado.

Aunque el análisis de los tipos de reformulaciones nos ha permitido comprender cómo se modelan las participaciones del niño y de los adultos en los dos tipos de intercambio, consideramos que resulta necesario expandir el análisis considerando específicamente los diferentes procedimientos de reformulación (Gülich \& Kotschi, 1997; Ciapuscio, 1997).

Tabla 3. Frecuencia relativa de uso de procedimientos de reformulación (\%) e índice de concentración de procedimientos según participantes y tipo de intercambio.

\begin{tabular}{|l|c|c|c|c|c|}
\hline $\begin{array}{l}\text { Participantes / Tipo de } \\
\text { intercambio: } \\
\text { (1. Entrevista } \\
\text { 2. Comentario grupal) }\end{array}$ & Verbalización & íCPR & Tratamiento & ICPR (t.) & $\begin{array}{l}\text { Calificación/ } \\
\text { evaluación }\end{array}$ \\
\hline 1. Niño & 0 & 0 & 100 & 0,58 & 0 \\
\hline 1. Adulto & 3 & 0,03 & 97 & 0,87 & 0 \\
\hline 2. Niño & 12,5 & 0,35 & 87,5 & 0,63 & 0 \\
\hline 2. Adulto & 12,5 & 0,37 & 87,5 & 0,41 & 0 \\
\hline
\end{tabular}

Como se observa en la Tabla 3, resulta llamativo que en ambos textos y tanto en las participaciones del niño como de los adultos, predominen los usos de procedimientos de tratamiento. Por otro lado, si comparamos los usos según los tipos de textos observamos que el adulto utiliza con mayor frecuencia procedimientos de tratamiento en el T1 $(0,87$ y 0,41; ICPR para T1 y T2, respectivamente), mientras que el niño presenta ICPR semejantes en ambos textos $(0,58 \mathrm{y}$ 0,63 , para $\mathrm{T} 1$ y $\mathrm{T} 2$, respectivamente) aunque varía notablemente el uso de procedimientos de verbalización ( 0 y 0,35 ; ICPR para T1 y T2, respectivamente). Por otro lado no se observa, en ningún caso, usos de procedimientos de calificación/evaluación metadiscursiva. 
Estos datos nos permiten suponer que, dado que los procedimientos de tratamiento suponen la existencia de una evaluación del hablante sobre el segmento tratado, en ambos intercambios los participantes procuran que en el texto se plasme esta actividad de monitoreo.

Con respecto a los procedimientos de verbalización, observamos tres usos ${ }^{5}$, uno en el T1 (del adulto) y en el T2 dos ocurrencias, una del niño y otra del adulto. A continuación transcribimos los procedimientos para poder compararlos.

T 1

66 Niño: Tengo una bolsa llenota / de dulces

67 Ent.: $\quad$ Ay me mu= (verbalización)

68 Niño: LGRAND-TO::TO::TA:

69 Ent.: ¿Cómo qué / de grande?

70 Niño: como > botan de basura< / eso que son grando::to::ta

71 Ent.: ¿Como esos botes de basura grandotes/ grandoto::tes/ llenos de dulces?

72 Niño: Sí:: / sí::

73 Ent.: ¡Ay me muero!/ Y: ¿yo cómo hago para conseguir uno?

74 Niño: $\quad{ }^{\circ}$ No sé ${ }^{\circ}$

El adulto en esta secuencia profiere un enunciado (l.67) incompleto, Gülich y Kotschi (1996) clasifican este tipo fenómenos como 'falsos comienzos'. Por nuestra parte, creemos, además que estos fenómenos representan la manifestación de un plan comunicativo paralelo ${ }^{6}$ que se descarta en una instancia del intercambio.

En este caso observamos que la expresión irrumpe en el turno del niño (ls. 66, 67 y 68: superposición), por lo que el entrevistador se percata de este hecho y ante la posibilidad de que el niño se amedrente y no prosiga con la formulación, la suspende. Pero, por otro lado suponemos que la entonación y la manifestación de la función emotiva del enunciado ${ }^{7}$ funcionan como alicientes para que el niño continúe el relato. Creemos que el niño interpreta exitosamente estas pistas y por ello continúa la formulación del segmento, es decir, no cede el turno al entrevistador y en la línea 68 completa la descripción de "la bolsa de dulces": segmentando el morfema, alargando vocales del segmento final y acentuando toda la emisión.

En el T2 el adulto también formula un 'falso comienzo' (Gülich \& Kotschi, 1997).

T2

05 Niño: La masa la estiré así: /y entonces le puse arriba (de) la caja// y:aparecieron números

06 Docente: Y aparecieron nú=:/ y aparecieron números: o... ¿ ¿los hiciste vos/los números? 
El adulto inicia su participación con una repetición parcial de la formulación del niño pero, antes de finalizar el segmento, lo interrumpe. Probablemente ha ocurrido una perturbación entre el texto que están produciendo en la interacción y el texto mental del adulto. Como hemos señalado previamente, consideramos que el hecho de que a la docente no le resulte claro cómo fue el proceso por el cual el niño modeló los números genera la formulación de la corrección de l.6. Ahora bien, ¿por qué no registramos, en T1 o en T2, correcciones a verbalizaciones del niño, cuando este en sus formulaciones presenta problemas de adecuación al sistema fonológico del español estándar?

Por ejemplo:

T1

l.12 Hicimos una ru::shca ... (Por rosca)

T1

ls. 61-62 Ellos no querían entrar a la piñata //

estaban >adr:en-tro<// pero no querían entrar a la piñata

Creemos que en los intercambios analizados se ha privilegiado, como meta comunicativa, la progresión temática; puesto que, en ninguno de los dos textos, el adulto ha corregido las formulaciones del niño. Paralelamente observamos que en otros fragmentos de la entrevista el niño pareciera no tener conciencia del disturbio que ocasiona la realización fonética deficiente de sus expresiones y asume la corrección externa, en este caso, por ejemplo, la de un compañero.

(De fragmentos no seleccionados: T2)

01 Niño: $\quad$ Acá cruzás para acá: // ahí hay una antopinta/ ¿no?

02 Ent.: $\quad$ ¿Una?

03 Niño: $\quad$ Una antopinta / y te va de flente

04 Ent.: $\quad$ ¿Una estufita?

05 Otro compañero: ¡UNA U-TO-PISTA!

06 Ent.: ¡A::!/ ¡una auto-pissta!

07 Niño: $\quad \quad \quad \quad<<$ Sí> $\quad$ cruzás para a-cá

En este fragmento, el entrevistador interrumpe la formulación del niño con una repetición parcial (l.2); consideramos que el adulto identificó en la verbalización una perturbación mucho más crítica, porque esa formulación obstaculiza la progresión informativa: que el interlocutor no pueda identificar el objeto punto de referencia, en el trayecto descripto, es un hecho que atenta severamente contra la coherencia de la situación que están componiendo. El adulto, 
entonces, intenta salvar la dificultad: solicita una repetición al niño con una expresión de tratamiento (l.2): una repetición parcial que como señalan Gülich y Kotschi (1997) funciona como una instrucción dirigida al interlocutor para repetir el elemento que ha desviado la expectativa del hablante en relación con la claridad acústica, la coherencia y/o el grado de adecuación a la norma lingüística.

Luego que el niño comprende la instrucción y formula la respuesta (l.3), la dificultad continúa $y$, ante esto, el adulto pone a consideración del niño su interpretación en una formulación alternativa (l.4). Esta actividad es la que causa la intervención de otro participante que ofrece una verbalización más adecuada a la que procuró formular el niño (l.5); hecho que queda corroborado a partir de la interjección del entrevistador (l.6), que demuestra la coherencia de ese término para el texto mental co-construido (Rogoff \& Toma, 1997), y por otro lado porque el niño continúa con el relato interrumpido (l.7).

En cuanto a los procedimientos de tratamiento observamos que los datos de frecuencia de la Tabla 3 parecerían señalar que el niño estructura su participación en el T1, en comparación con el T2, con una mayor frecuencia de procedimientos, no obstante al comparar los ICPR observamos que no existen diferencias tan notorias. Creemos que estos datos, en apariencia contrapuestos, son el resultado de comparar la frecuencia de procedimientos y la cantidad de procedimientos por enunciado. Dado que la cantidad de enunciados del niño en T2 es mucho menor que la cantidad de T1 observamos diferencias entre el IPCR (t.) de ambos textos $(0,58$ y 0,63 , para T1 y T2, respectivamente). En este sentido creemos que se produce en el T2 un uso más concentrado de procedimientos que podría deberse a la necesidad de acaparar la atención del docente y del auditorio en tan breves minutos de charla.

Por otro lado, la observación cualitativa nos permite reconocer que gran parte de esos procedimientos son repeticiones o paráfrasis, y prácticamente no se observan disociaciones o correcciones. Este dato podría resultar orientativo acerca del hecho de que ambos tipos de intercambios se han organizado a partir de la relación de equivalencia entre los enunciados. Consideramos que la relación de equivalencia colabora en el mantenimiento de la coherencia, pero también habilita que los referentes puedan ser activados por varias formas, es decir, es un recurso que propicia la continuidad de la interacción.

Analizando en detalle los tipos de procedimientos de tratamiento observamos que en el caso del T1 el adulto hace uso de las repeticiones, como por ejemplo se observa en los fragmentos transcriptos.

16 Ent.: ¿ ¿Te acordás cómo fue / $M$.

17 Niño: $\quad$ Con piñata 
18 Ent.: ¿Con piña:ta?

19 Niño: De Gokku< zeta> / la piñata

32 Ent.: ¿ ¿ y mirá si te la agarraba otro chico?

33 Niño: Yo me la agarré // Dos - había / Me las agarré a las dos

34 Ent.: ¿Las dos? ¿una qué / era grande?

En estas secuencias se observa que el adulto retoma total o parcialmente las expresiones del niño, pero al modificar la curva melódica y el tono, modifica el acto de habla. Entonces, lo que en la intervención del niño es una aserción, en la emisión del adulto es una interrogación.

En el T2 la repetición del adulto (l.9), a diferencia de las utilizadas por el entrevistador en el $\mathrm{T} 1$, respeta la línea melódica y no incluye una modificación del acto de habla; por lo que podemos inferir que pareciera querer retomar la aserción del niño.

Niño [...] y: aparecieron números

09 D $\quad$ Y aparecieron nú=/. y aparecieron números. o::¿ los hiciste vos los números?

A diferencia de la estrategia identificada en el T1, pareciera que el adulto al encabalgar su expresión sobre la del niño, le disputa el turno de habla, asimilando las características de su enunciación con las del niño. Posiblemente este recurso se articule en función del auditorio, es decir, el locutor adulto 'teatraliza' su intervención para semejar ante el auditorio que la formulación que emite es propiedad del niño. Pero, al hallar una incongruencia entre el texto construido en la interacción y su propio conocimiento de la situación, realiza una pausa prolongada y modifica las características de la entonación asumiendo ante el auditorio la responsabilidad del enunciado o, haciendo explícito el movimiento de asunción de responsabilidad.

En ambos intercambios, la repetición funciona como un ítem que asegura la coherencia, que le indica al interlocutor que el texto previo ya ha sido negociado y que resulta común para ambos participantes (Jefferson, 1983).

Si atendemos al carácter de los procedimientos de tratamiento (Gülich \& Kotschi, 1997) del niño, en el T1 observamos que presentan rasgos parecidos a los que se describen en la siguiente secuencia:

[El entrevistador procura que el niño narre eventos sucedidos durante la celebración de su cumpleaños.]

16 Ent.: ¿ ¿Te acordás cómo fue / $M$.

17 Niño: $\quad$ Con piñata

18 Ent.: ¿Con piña:ta? 
19 Niño: De Gokku=< zeta> / la piñata

20 Ent.: ¿De qué?

21 Niño: De GOKKU- ZETA / / la piñata

22 Ent.: ¿De gokku / del dragon boll?

23 Niño: Sí:

24 Ent.: ¿Con la forma de goku?

25 Niño: ((Silencio. Gesto de afirmación solo con la cabeza))

En este fragmento el adulto le solicita una narrativa sobre los eventos que acontecieron en su cumpleaños. El niño responde lacónicamente con la mención de un elemento (l.17 "con piñata") que, como se observa en los turnos posteriores, ha generado una serie de eventos que resultan muy significativos para el niño (ver en Apéndice: ls. 30 a 56: pugna por el contenido de la piñata). La pregunta del entrevistador (l.18), puede ser clasificada como una repetición pues retoma la expresión del niño, aunque introduce un alargamiento vocálico y modifica el acto de habla atendiendo a que el niño expanda esa descripción. El niño expande su propia formulación: menciona el personaje que permite identificar a la piñata (l.19). Esta última formulación genera un problema: el adulto parece no comprender esa mención (l.20), posiblemente porque el niño ha articulado la denominación del personaje con una segmentación para acelerar el ritmo (GOKKU<zeta>). En l.21 el niño produce una repetición, pero con una articulación más cuidada: omite la aceleración, sube el tono y marca con pausas más prolongadas la denominación del personaje. No obstante, en los turnos siguientes (l.23 y l.24) no puede especificar ni expandir esa mención. En este sentido las 'Autorreformulaciones' infantiles del T1 observan este patrón, muchas veces son repeticiones o expansiones mínimas de la formulación inicial.

Por otro lado, al analizar el funcionamiento de los procedimientos de tratamiento, observamos que poseen un eje de organización conceptual, una expresión que continuamente se expande, parafrasea y repite:

"Yo me agarré" (Cfr. ls. 31 a 38, 40 a 46, 50 a 58 y 76).

Este 'eje' se retoma en el procedimiento de resumen que cierra esta secuencia del T1:

Yo estaba agarrando //y yo llevaba esas bolsas / y ellos no venían / y yo metí en la bolsa la piñata// y la rompieron /y yo la rompí /y me agarré todo

Creemos que, con las expansiones y paráfrasis, el niño actualiza una estrategia en la que construye una imagen de sí mismo como ser 'desafiante', 'acaparador', en algunas circunstancias 'egoísta' o 'rebelde'; pero ante todo se constituye como un ser 'muy activo' frente a un medio que no reacciona ante sus acciones 0 , como ocurre con la enumeración de las actividades de los adultos, resultan remisos a participar. (ls. 30-32, ls. 40-43 y ls. 58-60). 


\section{Discusión y conclusiones}

Los resultados de este trabajo nos permiten reconocer cómo las diferencias en los formatos de interacción influyen en el uso infantil de ciertos recursos lingüísticos.

De esta forma podemos hipotetizar, que en el T1, tanto el niño como el adulto, identifican la función que a cada uno de ellos le corresponde: el niño es el informante y el adulto el que interroga, y que la división del trabajo resulta, en este sentido, estricta y muy asimétrica. Este hecho se manifiesta claramente en la inexistencia de Reformulaciones Externas.

En cambio, en el T2 los participantes comprenden que el texto también se está gestando para un auditorio, por lo que ya no existe una distancia tan estricta en la división de tareas: 'aquel que informa' y 'el que indaga'; sino que 'el que informa' también debe controlar que la versión reformulada por los enunciados del adulto resulte una versión consensual. Pareciera que los participantes se preocupan para que, en el momento del intercambio, quede claro- incluso para el auditorio- que el texto final es fruto de negociaciones.

Por otro lado, hemos observado que en el T1 además de negociarse contenidos conceptuales se negocian imágenes y valoraciones acerca de las conductas normativas (y esperables) del anfitrión del cumpleaños. A este respecto, Jefferson (1983) considera que las reformulaciones, en tanto manifiestan la capacidad de negociar contenidos de los participantes, son un recurso fundamental para construir o fortalecer identidades en el intercambio comunicativo. Consideramos que en este caso, además, los dos participantes del intercambio manejan las pautas del formato, concretamente que en una entrevista no solo se narran hechos sino que se exponen valoraciones, opiniones y circunstancias que suponen la imagen de cada uno de los participantes del intercambio.

En función de estas consideraciones, estimamos que los usos infantiles de procedimientos de reformulación pueden compararse a los usos adultos, pues forman parte del grupo de recursos que manifiestan las estrategias de construcción, de afirmación de identidad/de pertenencia a un grupo (van Dijk, 1996; Courtis, 2000).

Además, en lo que atañe a la entrevista (T1), hemos observado la preeminencia de repeticiones, expansiones y paráfrasis, hecho que nos permite suponer que este tipo de interacción, en tanto texto mental compartido (Rogoff \& Toma, 1997), se organiza estructuralmente a partir de la relación de equivalencia-expansión entre los enunciados. La relación de equivalencia colabora en el mantenimiento de la coherencia, pero también habilita que los referentes puedan ser activados por varias formas, es decir, es un recurso que propicia la continuidad de la interacción y crea un texto mental compartido conceptualmente más rico. 
Por otro lado, no hemos hallado en ninguno de los dos intercambios procedimientos de calificación (Gülich \& Kotschi, 1997). Si tomamos en cuenta que Brandt y Rosengren (1992) consideran que los procedimientos de calificación operan sobre actos de habla, y que estos resultan, en relación con el ejercicio de la función de monitoreo, más complejos que los procedimientos de verbalización y tratamiento (Silva, 2007), podemos establecer algunas hipótesis sobre los factores que han ocasionado esa ausencia.

Estimamos que la hipótesis más adecuada es la que refiere a la edad de uno de los participantes del intercambio. En efecto, el hecho de que el niño sea tan pequeño condicionaría la selección de procedimientos de calificación, pues estos procedimientos son actividades complejas que exigen que el hablante, además de la reflexión acerca del tipo de desviación, pueda retener un segmento importante del intercambio y además active una forma alternativa que justifique la solicitud de excusamiento (Gülich \& Kotschi, 1997). En este sentido, si el adulto utilizara alguna de estas formas estaría demandando del niño la posibilidad de que, también, recupere un segmento considerable del intercambio (un acto de habla) y, asimismo, comprenda el carácter del excusamiento que la forma actualiza.

En suma, el análisis aquí presentado nos permite concluir que en el diferente uso y frecuencia de ‘Autorreformulaciones' en el T1 y de Reformulaciones Externas en el T2 se manifiestan diferencias en la gestión del control. Asimismo, este fenómeno nos indica que tanto el niño de 5 años como los adultos configuran sus interacciones en virtud de las exigencias del formato.

Por otro lado, el análisis de la organización de los procedimientos de reformulación ha permitido explorar la relación compleja que existe entre la actividad cognitiva de control sobre el texto en gestión y ciertas características de la dinámica comunicativa, específicamente las exigencias del formato y las diferencias evolutivas de los participantes.

Finalmente, creemos que para que estas observaciones posean mayor grado de validez resulta necesario complementar este tipo de análisis con investigaciones que, con una muestra considerable de sujetos, establezcan cuáles son las tendencias de los procedimientos de reformulación en los dos tipos de texto considerados.

\section{REFERENCIAS BIBLIOGRÁFICAS}

Anselmi, D., Tomasello, M. \& Acunzo, M. (1986). Young children's responses to neutral and specific contingent queries. Journal of Child Language, 13, 135-144.

Brandt, M. \& Rosengren, I. (1992). Sobre la estructura ilocucionaria del texto. Lili, 2, 9- 51. 
Ciapuscio, G. (1997). Los científicos explican: La reformulación del léxico experto en la consulta oral. Cuadernos do Instituto de Letras, 18, 36-46.

Cole, M. (1999). Psicología cultural. Madrid: Morata.

Courtis, C. (2000). Construcciones de alteridad: Discursos cotidianos sobre la inmigración coreana en Buenos Aires. Buenos Aires: Eudeba.

Erickson, F. (1996). Going for the zone: The social and cognitive ecology of teacher-student interaction in classroom conversations. En D. Hicks (Ed.), Discourse, Learning and Schooling (pp. 30-62). Cambridge, New York: Cambridge University Press.

Erickson, F. (2004). Talk and social theory. Malden, M. A.: Polity.

Garret, M. (1988). Procesos en la producción del lenguaje. En F. Newmeyer (Comp.), Panorama de la Lingüística Moderna. El lenguaje: Aspectos psicológicos y biológicos (pp. 91-121). Madrid: Visor.

Garvey, C. (1977). The contingent query and dependent act in conversation. En M. Lewis \& L. Rosenblum (Eds.), Interaction Conversation and the Development of Language (pp. 6393). New York: Wiley.

Gülich, E. \& Kotschi, T. (1987). Les actes de reformulation dans la consultation. La Dame de Caluire. En P. Bange (Ed.), L'analyse des interactions verbales. La dame de Caluire: Une consultation. Actes du Colloque tenu a l'Université Lyon 2 du 13 au 15 decémbre 1985 (pp. 15-81). Berna: Peter Lang.

Gülich, E. \& Kotschi, T. (1997). Discourse production in oral communication: A study based on French. En U. Quastoff (Comp.), Aspects of Oral Communication (pp. 31-66). Berlin: Mouton de Gruyer.

Gumperz, J. (1982). Discourse strategies. Cambridge: Univ. Press.

Hunt, K. (1970). Recent measures in syntactic development. En M. Lester (Ed.), Reading in applied transformational grammar (pp. 179-192). Nueva York: Holt, Rinehart and Wiston.

Jefferson, G. (1983). On exposed and embedded correction in conversation. En G. Button \& J. Lee (Eds.), Talk and Social organization (pp. 86-100). Clevedon: Multilingual Matters.

Jefferson, G. (2004). Glossary of transcript symbols with an Introduction. En G. Lerner (Ed.), Conversation Analysis: Studies from the first generation (pp.13-23). Philadelphia: John Benjamins.

Lloyd, P. (1991). Strategies used to communicate route directions by telephone: A comparison of 7-year-old, 10-year- old, and adults. Journal of Child Language, 18, 171-190.

Macbeth, D. (2004). The relevance of repair for classroom correction. Language and Society, 33, 703-736. 
Michaels, S. (1988). Presentaciones narrativas: Una preparación oral para la alfabetización con alumnos de primer curso. En J. Cook-Gumperz (Comp.), La construcción social de la alfabetización (pp.109-136). Barcelona: Paidós.

Newell, A. \& Simon, H. (1972). Human problem solving. Englewood Cliffs, NJ: Prentice Hall.

Pomeranz, A. \& Fehr, B. J. (2001). Análisis de la conversación: Enfoque del estudio de la acción social como prácticas de producción de sentido. En T. van Dijk (Ed.), El discurso como interacción social (pp. 101-140). Barcelona: Gedisa.

Rogoff, B. \& Toma Ch. (1997). Shared thinking: Community and institutional variations. Discourse Processes, 23(3), 471-497.

Rosemberg, C. (2002). La conversación en el aula. Aportes para una teoría de la enseñanza a través del discurso. Tesis doctoral inédita. Buenos Aires: Facultad de Filosofía y Letras, Universidad de Buenos Aires.

Rosemberg, C. \& Manrique, M. S. (2004). Relatos compartidos en la interacción entre la maestra y los niños en el jardín de Infantes. Ponencia presentada en el II Coloquio Argentino de la IADA, International Association for Dialogue Analysis, Universidad de La Plata.

Rosemberg, C. \& Manrique, M. S. (2005). Compartir experiencias personales en la escuela infantil. Un medio para reconstruir lingüísticamente la vida de los niños. Ponencia presentada en las IV Jornadas de Desarrollo Humano y Educación, Universidad de Alcalá de Henares, España.

Rosemberg, C. \& Silva, M. L. (2009). Teacher children interaction and concept- development in kindergarten. Discourse Processes, 46(6), 572-591.

Schegloff, E., Jefferson, G. \& Sacks, H. (1977). The preference for self-correction in the organization of repair in conversation. Language, 53, 361-382.

Schwe, H. \& Markman, M. (1997). Young children appreciation of the mental impact of the communicative signs. Developmental Psychology, 33, 630-635.

Silva, M. L. (2007). Hacia una sistematización de los mecanismos de formulación en función de la teoría Sociocultural. Estudios de Psicología, 28(1), 33-49.

Silva, M. L., Plana, D. \& Borzone, A. (2007). Representaciones mentales de eventos y habilidades discursivas en niños pequeños. Ponencia presentada en el III Coloquio Argentino de la IADA, International Association for Dialogue Analysis, Universidad de La Plata.

Silvestri, A. (1999). Bajtín y Vygotski: Teoría del enunciado y concepción socio-cultural del psiquismo. En P. del Rio (Comp.), Explorations in Socio-cultural Studies: Historical and Theoretical Discourse (pp. 214-219). Madrid: Infancia y Aprendizaje.

Tomasello, M., Farrar, J. \& Dines, J. (1983). Children speech revision for a familiar and unfamiliar adults. Journal of Speech and Hearing Research, 27, 359-363. 
van Dijk, T. (1996). Discourse, racism and ideology. La Laguna: RCEl ediciones.

Véliz, M. (1999). Complejidad sintáctica y modo del discurso. Estudios Filológicos, 34, 181-192.

Vygotsky, L. (1961). Thought and speech. En S. Saporta (Ed.), Psycholinguistics: A book of readings (pp. 197-260). New York: Holt, Rinehart and Winston.

Vygotsky, L. (1993). Pensamiento y lenguaje: Teoría del desarrollo cultural de las funciones psíquicas. Buenos Aires: Fausto.

\section{NOTAS}

1 La problemática lingüística identificada por los docentes posiblemente se deba a que la mayor parte de los niños se encuentra en una situación de contacto lingüístico, porque el registro de habla que utilizan no coincide con el perfil de un hablante de su misma edad de clase media, y/o porque poseen un contacto poco formal con material escrito y eventos de lectura antes del ingreso a la institución educativa.

2 Se han utilizado las siguientes codificaciones:

¿X? Entonación ascendente para interrogaciones

¡X! Entonación ascendente para exclamaciones

MAYÚSCULA Volumen alto o aumento de volumen

.XX. Descenso de volumen o volumen bajo

XX Énfasis

/ micropausa intraturno

// pausa corta intraturno

/// pausa larga intraturno

( silencio) pausa larga interturno

$<. X X>$. Emisiones rápidas

$>X X<$. Emisiones lentas

se-pa-ra-ción Separación rítmica de sílabas

.CCC Superposición de emisiones o de acciones no verbales y emisiones

: Vocal prolongada

::: Incremento en la prolongación de la vocal

XX Prolongación de consonante

(( XXX)) Acción no verbal

Otras codificaciones

M. Niño entrevistado

ENT Entrevistador

D Docente

Superíndice Irrupción de otro interlocutor no previsto en el intercambio

CC Nombre de otro niño que participa del intercambio

$\{X X\}$ Comentarios del transcriptor.

3 Se ha aclarado entre paréntesis y con subíndice el tipo de procedimiento, para ello se utilizan la inicial del tipo de procedimiento (v.: verbalización, t: tratamiento y c: calificación). 
$4 \quad$ No se presenta el ICPR(c.) pues no se registran usos.

5 No hemos relevado aquí las ocurrencias de fenómenos de verbalización que corresponden al nivel pragmático (duración de las pausas, variaciones en la curva melódica, disminución o incremento del ritmo, etc.), ya que para Gülich y Kotschi (1997) estos procedimientos orientan acerca del tipo de empaquetamiento de unidades de información y consideramos que este dato resulta poco operativo, en función de los objetivos del trabajo.

6 Este tipo de fenómenos también ha sido reconocido, entre otros, por Garret (1988), cuando propone su modelo de producción de habla. En consonancia con los planteos de Gülich y Kotschi (1997), Garret (1988) atribuye la causa del fenómeno a una 'elección semántica y/o comunicativa descartada'.

7 En el dialecto rioplatense la expresión: ‘¡Ay, me muero!' con entonación marcadamente ascendente y acompañada de gesticulación característica se interpreta en forma equivalente a un: '¡Qué fantástico!, quisiera compartir eso'.

8 Se han subrayado con línea simple las formulaciones del niño y con línea doble las repeticiones del adulto.

\section{APÉNDICE}

01 M Cinco m: hay allá

02 ENT. ¿Cuántos años tenés/ Miguel

03 M Seis

04 ENT. ¿Cuándo los cumpliste?

$05 \mathrm{M}^{\circ} \mathrm{En}^{\circ}$ febrero

06 ENT. En febrero// ¿los cumplís?

07 M En marzo / digo

08 ENT. Y: ¿te hicieron un cumpleaños/ M.?

09 M ((Gesto afirmativo solo con movimiento de la cabeza))

10 [[Irrumpe Marvin: A mí también en Bolivia/ me hicieron un cumpleaños// ahora ya tengo/ ahora cinco añoss

$11 M_{\text {¿Y qué? }}$

12 ENT. ((a M. )) ¿Cómo se llama?

$13^{\circ}$ Marvin $^{\circ}$

14 M. está grabando

15 ENT. Sí / pero ahora nos callamos porque M. nos está contando cómo fue su cumpleaños]]

16 ENT. ¿Te acordás cómo fue / $M$.

17 M Con piñata

18 ENT. ¿Con piña:ta?

19 M De Gokku< zeta / la piñata

20 ENT. ¿De qué?

21 M De Gokku zeta / la piñata

22 ENT. ¿De gokku / del dragon boll?

23 M Sí: 
24 ENT. ¿Con la forma de goku?

25 ((Silencio. Gesto de afirmación solo con la cabeza, gira la cabeza hacia donde estaban jugando sus compañeros, flexiona el cuerpo))

26 ENT. ¿Vos la pedisste?

27 M ((Silencio Gesto afirmativo con la cabeza se agacha para tomar un autito))

28 ENT. ¿Y qué hiciste/ con la piñata

29 M ((Se incorpora y mira a la entrevistadora)) La rompí y todos se agarraron//

$30<$ Yo me agarré> una pistola grande (( señala una dimensión ensanchando la distancia entre las palmas))/que era así de tres plazas / de agua //de agua / esa cayó de la piñata // 31 Yo me LA- AGARRÉ

32 ENT. ¿y mirá si te la agarraba otro chico?

33 M Yo me la agarré // Dos - había / Me las agarré a las dos

34 ENT ¿Las dos? ¿una qué / era grande?

35 M Una era más grande y una era un poco más chiquita

36 ENT Otra más chiquita / y: ¿de qué color era?

37 M Rojo y <uno azul>

38 ENT ¿Y cuál vos te agarraste

$39 \mathrm{M} \mathrm{La}$ roja /y la azul

40 ENT Las dos para vos / y los otros chicos< no te decían nada>

$41 \mathrm{M}$ ¡Nada!

((.Gesto de negación solo con la cabeza, hacia un lado y hacia el otro))

42 . $<$ No agarraron ninguna $>/<$ yo me agarré todos los ${ }^{\circ}$ Chiches $^{\circ}>$ y ellos NADA

43 ENT ¿Nada? / ¿no se= enojaron?

$44 \mathrm{MNO}$

45 ENT ¿No?

$46 \mathrm{M}$ Yo me agarré TODO

47 ENT Y: ¿había otrass cosass?

$48 \mathrm{M}^{\circ} \mathrm{S}^{\prime}{ }^{\circ} / /$ Yo tenía una piñata de goku zeta/ y de digimon/ en la digimon había muchas

cosas

49 ENT ¿Qué cosas/ había en la de digimon?

50 M Chocolates// dulce/ cigarro/ para mi mamá

51 ENT ¿También para tu mamá?

$52 M$ Y yo me agarré toda la cosa

53 ENT ¿Las de tu mamá/ también?

54 M ((Gesto de afirmación solo con la cabeza) Sí / les dí la poca de señoress

55 ENT ¿En serio? \{cambia timbre y calidad de voz como si estuviera riendo\}

$56 \mathrm{M} \mathrm{Me}$ agarré muchas cosas// ellos tampoco/ nada/ también

57 ENT ¡Pero eso no se hace M.! // cómo te vas a agarrar todo! \{el mismo tono de enunciado 55$\}$

58 M Sí / yo me agarré todo

59 ENT ((Risas)) Ellos estaban mirando/ y vos / /agarraste las cosas=

60 ENT . $=<$ Porque $>$ ellos no querían entrar

$61 \mathrm{M}$. .Ellos no querían entrar a la piñata //

62 M. estaban .adren-tro.// pero no querían entrar a la piñata 
63 ENT ((a otro chico que le muestra un juguete)) .¡Qué lindo CC una pistola!

64 .Ellos estaban mirando

65 ENT Y.. entonces.. ahora tenés una pistola de agua /y los- dulcess

66 M. Tengo una bolsa llenota / de dulces

67 ENT . Ay me mu=

68 .=GRANDO-TO::TO::TA:

69 ENT ¿Cómo qué / de grande?

$70 \mathrm{M}$. como . botan de basura. / eso que son grando::to::ta

71 ENT ¿Como esos botes de basura grandotes/ grandoto::tes/ llenos de dulces?

72 M. Sí:: / Sí::

73 ENT ¡Ay me muero!/ Y: ¿yo cómo hago para conseguir uno?

$74 \mathrm{M}$. . No sé.

75 ENT ¿Cómo hiciste vos?

76 M. Yo estaba agarrando //y yo llevaba esas bolsas / y ellos no venían / y yo metí en la bolsa la piñata// y la rompieron /y yo la rompí /y me agarré todo

T2 Momento comentario grupal

$01 \mathrm{D}$ ¿A qué: jugaste?

$02 \mathrm{M}$. Yo jugué a la masa

$03 \mathrm{D}$ Vos jugaste con la masa y: ¿qué hiciste con la masa?

04 M. ¡PLAS $<$ TILINA $>$ !

$05 \mathrm{D}$ Y: ¿qué hicimos con la plastilina?

06 M. Hicimos una ru::shca //. y había una ruta gigante

$07 \mathrm{D}$ ¡Mirá qué: bien!/ y:: vos/¿qué hiciste?

$08 \mathrm{M}$. La masa la estiré así: /y entonces le puse arriba (de) la caja// y: aparecieron números

$09 \mathrm{D} Y$ aparecieron nú=/. y aparecieron números. o::¿los hiciste vos los números?

$10 \mathrm{M}$. Solamente aparecieron números /.porque la caja tenía números. 